\title{
Short-Term Recanalization of Symptomatic Internal Carotid Artery Occlusion
}

\author{
Hyun Jo Lee, MD, Seungyoo Kim, MD, Yoon Sang Oh, MD, PhD, Woojun Kim, MD, PhD, \\ and A-Hyun Cho, MD, PhD \\ Department of Neurology, Yeouido St. Mary's Hospital, Catholic University of Korea, College of Medicine, Seoul, Korea
}

\begin{abstract}
Background: The natural history of acute symptomatic ICA occlusion remains unclear. Short term follow-up studies have been rarely reported. Therefore, the objective of this study was to determine the natural history of acute ICA occlusion through short-term follow-up. In addition, we determined factors associated with recanalization and poor outcome.

Methods: We consecutively enrolled acute ischemic stroke patients within 7 days who had acute symptomatic internal carotid artery occlusion. Demographic data, stroke subtypes, National Institute of Health Stroke Scale (NIHSS) score, and modified Rankin scale score at 3 months were recorded. Carotid duplex ultrasonography, CT angiography, or digital subtraction angiography in 2-9 days after the initial angiography was conducted to check recanalization pattern of ICA. Recanalization was classified into complete, partial, or no recanalization.

Results: A total of 64 patients with acute symptomatic ICA occlusion were enrolled in this study. Follow-up vessel studies were completed for 53 patients. Follow-up carotid duplex sonography was performed for 23 patients. CT angiography was performed for 9 patients. Both carotid duplex sonography and CT angiography were performed for 21 patients. Complete recanalization was observed in $5(9.4 \%)$ of the 53 patients. All five patients received thrombolysis. Partial recanalization was observed in $8(15.1 \%)$ patients. The remaining 40 (75.5\%) patients did not show recanalization. Cardioembolism $(P=0.008)$ and thrombolytic treatment $(P=0.025)$ were factors associated with complete recanalization. However, recanalization $(P>0.999)$ was not associated with favorable clinical outcome.

Conclusion: Recanalization of symptomatic internal carotid artery occlusion was identified in 13 (24.5\%) patients. Cardioembolism and thrombolytic treatment were factors significantly associated with complete recanalization.

J Neurocrit Care 2015;8(2):103-108
\end{abstract}

Key Words: Internal carotid artery diseases; Angiography; Duplex ultrasonography

\section{INTRODUCTION}

Occlusion of internal carotid artery (ICA) accounts for $6-15 \%$ of all acute cerebrovascular events. ${ }^{1}$ The outcome of patients with acute occlusion of the ICA is rather poor, with disability historically reported at $40 \%$ to $69 \%$ and a $16 \%$ to $55 \%$ mortality rate. ${ }^{2,3}$ Recanalization of the ICA after an acute occlusion - either spontaneous or possibly induced by thrombolysis - has been reported and usually occurs early, between 6 hours and 2 weeks after the event. ${ }^{4-7}$ But there is limited data on the frequency, time course, stroke mechanisms and clinical

Received: August 27, 2015 / Revised: September 17, 2015

Accepted: September 18, 2015

Address for correspondence: A-Hyun Cho, MD, PhD

Department of Neurology, Yeouido St. Mary's Hospital, Catholic University of Korea, College of Medicine, 10, 63-ro, Yeongdeungpogu, Seoul 07345, Korea

Tel: +82-2-3779-2433, Fax: +82-2-782-8654

E-mail: ahyun@catholic.ac.kr outcome involved in recanalization with short term follow-up studies. We aimed to investigate the natural history of acute ICA occlusion through short-term follow-up and the factors which are associated with recanalization and poor outcome.

\section{METHODS}

\section{Patients}

We consecutively enrolled acute ischemic stroke patients within 7 days, in whom acute symptomatic internal carotid artery occlusion was observed. The ischemic stroke was diagnosed with diffusion-weighted MRI and clinical examination. The ischemic stroke lesion should be relevant to ICA occlusion. We obtained the demographic data (age, sex, risk factors), previous medical history, etiology of stroke, acute management, National Institute of Health Stroke Scale (NIHSS) score, modified Rankin scale score at 3 months after the stroke. Modified Rankin scale was obtained through telephone interview 

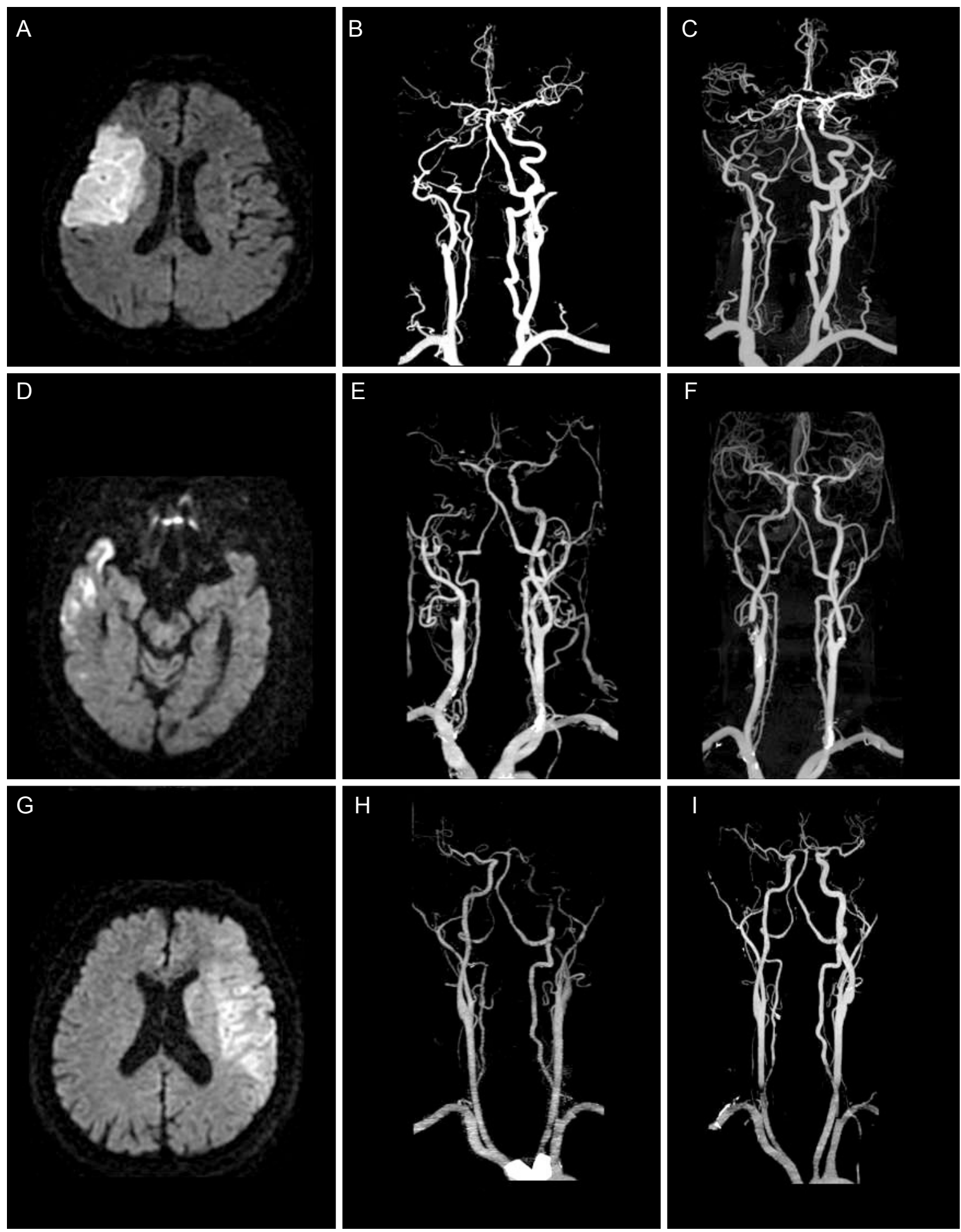

Figure 1. Three cases of acute symptomatic ICA occlusion. Initial diffusion-weighted MRIs (left column), initial CTA images (middle column) and follow up CTA images (right column). The first row: A 71-yo-male presented with left facial palsy and left arm weakness. DWI shows acute infarction in the right MCA territory (A). The right proximal ICA occlusion was not recanalized after 6 days $(B, C)$. He was treated with antiplatelet due to his initial NIHSS was 3(minor symptoms). The second row: A 83-yo-female presented with right hemiparesis (Initial NIHSS 13). Initial brain images showed right MCA territory infarction with occlusion of right ICA from carotid bifurcation (D, E). Follow-up examination on day 7 after thrombolysis shows partial recanalization of right proximal ICA (F). The patient was discharged with improved neurological situation (NIHSS 5, MRS 1). The third row: A 65-yo-male presented with global aphasia and right side weakness. Acute left MCA territory infarction is observed $(\mathrm{G})$. Complete recanalization of left distal ICA occlusion was observed after 5 days (H, I). Stroke subtype was cardioembolism due to paroxysmal atrial fibrillation. He was treated with IV tPA and IA thrombolysis and mRS at 3 months was 2. 
or outpatient clinic exam. As stroke work-ups, routine blood tests, chest X ray, electrocardiography, echocardiography, 24hour Holter monitoring were performed. Stroke subtype was defined according to the TOAST classification. ${ }^{8}$

\section{Imaging protocol}

The stroke MRI protocol consisted of a diffusion-weighted image, a gradient echo image, a fluid-attenuated inversion recovery image, and a T2-weighted image. CT or MR angiography was also performed as a routine vessel work-up. Helical (3D) CT angiography was performed with a LightSpeed VCT (General Electric Medical System, Milwaukee WI, USA). A three-dimensional CT angiogram was made with a computer workstation (Rapidia, Infinitt). The MR angiography parameters included a flip angle of $20^{\circ}$, a matrix number of $320 \times 224$, and a field-of-view of $200 \times 200$. Three-dimensional contrastenhanced MR angiography from the aortic arch to the level of the central skull base was obtained using an intravenous bolus injection of $15 \mathrm{~mL}$ gadopentetate dimeglumine with a repetition time of $27 \mathrm{msec}$ and an echo time of $6.9 \mathrm{msec}$. We further conducted carotid duplex ultrasonography, CT angiography or digital subtraction angiography in 2-9 days after the initial angiography to check recanalization pattern of ICA.

\section{Interpretation of data}

Recanalization was classified into complete, partial, and no recanalization. Complete recanalization was defined when there was no remained luminal stenosis. Partial recanalization was defined when focal luminal stenosis was remained. No recanalization was defined when there was no change from the initial study (Fig. 1). To minimize the possibility of misinterpreting the pseudo-occlusion as complete occlusion, the axial images of the CTA and the reconstructions were carefully reviewed.

\section{Statistical analysis}

A descriptive analysis was done for frequency data. To compare characteristics between patients with recanalization and those without, we used Mann-Whitney test for continuous variables, Fisher's exact test and Chi-square test for categorical variables. SPSS software for Windows version 13.0 (SPSS Inc., Chicago, IL, USA) was used. This study was approved by institutional review board of our hospital. An informed consent was not obtained because this study was retrospectively performed.

\section{RESULTS}

\section{Baseline characteristics}

Between June 2009 and July 2014, a total of 64 patients with acute symptomatic ICA occlusion were consecutively included. $32(50 \%)$ were males, and their mean age was $73.16 \pm 10.35$ (SD). Of the 64 patients, 11 patients could not perform followup vessel studies due to poor neurological condition $(n=5)$ or death $(n=6)$. Therefore, $53(82.8 \%)$ patients were included in the analysis. The median time of follow-up was 3 days (interquartile range, 2 to 5). Carotid duplex sonography as a follow-up examination was done in 23 patients and CT angiography in 9 and both of them in 21. The most common stroke mechanism was large vessel diseases $(n=34)$, followed by cardioembolism $(\mathrm{n}=17)$ and other causes (cryptogenic, dissection, $\mathrm{n}=2$ ). 15 patients received intravenous thrombolysis (one had additional intra-arterial thrombolysis), 37 patients received antiplatelet treatment and one patient was treated with anticoagulation (low molecular weight heparin) as an acute treatment. Among the 15 patients with thrombolysis, recanalization was observed in four.

\section{Recanalization}

Complete recanalization was observed in 5 (9.4\%), all of whom were cardioembolic stroke and four patients received intravenous thrombolysis. Partial recanalization was observed in $8(15.1 \%)$ patients. Eventually, $40(75.5 \%)$ patients did not show recanalization. Cardioembolism $(P=0.008)$ and thrombolytic treatment $(P=0.025)$ were significantly associated with the complete recanalization (Table 1).

\section{Clinical outcome and hemorrhagic transformation}

Regarding clinical outcome, mRS could be obtained in 51 patients. Among them, 23 (43.4\%) patients had favorable clinical outcome. However, complete recanalization was not associated with the favorable clinical outcome $(P>0.999)$. Fortytwo patients underwent a follow-up brain CT scan to look for hemorrhage as a part of our routine follow-up or if there was clinical deterioration. Hemorrhagic transformation (symptomatic 1 and asymptomatic 2) was observed in 3 patients, all of 
whom were those with recanalized (complete or partial) internal carotid artery.

\section{DISCUSSION}

While not a frequent phenomenon, several reports have documented small numbers of patients with late spontaneous recanalization of ICA occlusions. Most prior studies have focused on chronic ICA occlusion with long term follow-up., ${ }^{9,10}$ They found that most probable etiology of the occlusion was atherosclerotic plaque and partial recanalization was produced by spontaneous lysis. Sometimes recanalization of the occluded internal carotid is heralded by a disabling neurologic deficit and a thromboembolic occlusion of other arteries. ${ }^{10,11}$ However, a retrospective study of eight patients with carotid artery occlusion showed that spontaneous late recanalization was seen in six patients with few or no clinical sequelae. ${ }^{12}$

Our study was designed to evaluate early recanalization rates of the occluded ICA in acute stroke patients. To date, only a few scattered reports have documented follow-up data about acute symptomatic ICA occlusion. ${ }^{5,9,10}$ Our study has several strengths. First, our subjects are prospectively included 64 consecutive patients with 3-month clinical outcome. Second, most patients underwent follow-up study by means of the carotid duplex scans or the CTA. Finally, the endovascular treatment was not performed in all our subjects. This finding suggests more natural recanalization history of acute ICA occlusion in our real clinical field.

In our $13(24.5 \%)$ cases of acute ICA occlusion leading to ischemic stroke, subsequent vessel reopening was observed between the second and ninth day after the initial angiography. This is a little higher rate than the previous study which showed $15.8 \%$ of recanalization. ${ }^{5}$ The discrepancy is probably due to a different population and different treatment protocols for acute ICA occlusion between those two studies.

Early recanalization was more complete in patients with cardioembolic stroke compared with other stroke subtypes. This data indicate that mechanisms of vessel occlusion are

Table 1. Clinical characteristics and outcome according to the recanalization patterns

\begin{tabular}{|c|c|c|c|c|c|}
\hline & \multirow{2}{*}{$\begin{array}{l}C R \\
n=5\end{array}$} & \multicolumn{3}{|c|}{ Non-CR } & \multirow{2}{*}{$P$-value } \\
\hline & & Total, $\mathrm{n}=48$ & $P R, n=8$ & $N R, n=40$ & \\
\hline Age & $68.4 \pm 6.0$ & $73.3,8.6$ & $74.1,10.7$ & $73.1,8.2$ & 0.192 \\
\hline Male & $1(20.0)$ & 28 (58.3) & $5(62.5)$ & $23(57.5)$ & 0.164 \\
\hline Hypertension & $3(60.0)$ & $40(83.3)$ & $7(87.5)$ & $33(82.5)$ & 0.235 \\
\hline DM & $2(40.0)$ & $16(33.3)$ & $3(37.5)$ & $13(32.5)$ & $>0.999$ \\
\hline Dyslipidemia & $3(60.0)$ & $20(41.7)$ & $5(62.5)$ & $15(37.5)$ & 0.642 \\
\hline Smoking & $1(20.0)$ & $12(25.0)$ & $2(25.0)$ & $10(25.0)$ & $>0.999$ \\
\hline Initial NIHSS (median, IQR) & $14(10-19)$ & $7(3-13)$ & $13.5(7.25-16.75)$ & $5.5(2.25-12.75)$ & 0.04 \\
\hline Follow-up study & & & & & 0.065 \\
\hline CT angiography & $2(40.0)$ & $7(14.6)$ & $2(25.0)$ & $5(12.5)$ & \\
\hline Sonography & 0 & $23(47.9)$ & $1(12.5)$ & $22(55.0)$ & \\
\hline Both & $3(60.0)$ & $18(37.5)$ & $5(62.5)$ & $13(32.5)$ & \\
\hline Thrombolysis & $4(80.0)$ & $11(22.9)$ & $5(62.5)$ & $6(15.0)$ & 0.025 \\
\hline TOAST subtype & & & & & 0.008 \\
\hline LVD & $0(0)$ & $34(70.8)$ & $5(62.5)$ & $29(72.5)$ & \\
\hline Cardioembolism & $5(100.0)$ & $12(25.0)$ & $2(25.0)$ & $10(25.0)$ & \\
\hline Others & $0(0)$ & $2(4.2)$ & $1(12.5)$ & $1(2.5)$ & \\
\hline Favorable outcome (mRS 0-2) & $2(40.0)$ & $21(45.7)$ & $4(50.0)$ & $17(42.5)$ & $>0.999$ \\
\hline Hemorrhagic transformation & $2(40.0)$ & $1(2.1)$ & $1(12.5)$ & $0(0)$ & 0.033 \\
\hline
\end{tabular}

Values are number of patients (\%) or mean \pm SD unless otherwise indicated.

CR, complete recanalization; PR, partial recanalization; NR, no recanalization; SD, standard deviation; DM, diabetes mellitus; NIHSS, national institute of health stroke scale; IQR, interquartile range; LVD, large vessel disease; mRS, modified Rankin Scale; $p$ value suggests the results of comparison between $\mathrm{CR}$ and non-CR. 
thought to be relevant for the chance of recanalization. It might therefore be hypothesized that cardioembolic arterial occlusions might have the best chance of recanalization even total ICA occlusion with large clot burden. Also most cases of complete recanalization were observed in patients who received intravenous thrombolysis. This may suggest that intravenous thrombolytic therapy might facilitate vessel reopening in acute symptomatic ICA occlusion. However, we have to be prudent in this interpretation because this result was drawn by a univariate analysis.

Partial recanalization of the ICA occlusion was considered when the occluded vessel was partly recanalized allowing some residual flow improvement with a severe ICA stenosis or when the embolus moved and partly occluded a distal vessel. Other possible explanation for partial recanalization with distal occlusion is that the proximal vessels were never completely occluded but extremely slow flow by high-grade obstructions of the distal arteries. Strokes in these conditions are ascribed to partial resorption of distal ICA atherosclerotic thrombus, whereas distal migrations of embolic fragments discussed earlier are thought to be more common in cardioembolic stroke than other etiologies. ${ }^{13}$

Establishing the strength of the association between recanalization and clinical outcomes is critically important. It has often been suggested that recanalization might have a deleterious influence on tissue injury by promoting reperfusion injury, excessive cerebral edema and hemorrhagic conversion of the infarct. ${ }^{14}$ However, a prior meta-analysis of published data between 1985 and 2002 reported that recanalization is strongly associated with improved functional outcomes and reduced mortality. ${ }^{15}$ In our study, patients who experienced complete recanalization of an ICA were not related to early neurological deterioration and also not associated with a more favorable outcome compared with no recanalization group.

In conclusion, acute symptomatic ICA occlusion is now considered to be a dynamic process involving multiple systemic and local mechanisms. Serial assessment of recanalization of symptomatic ICA occlusion might be useful for identifying a stroke subtype and provide adequate evidence to guide acute treatment decision. It would also suggest that patients who experience partial recanalization with atherosclerotic pathology might be reasonable candidates for a carotid endarterectomy or carotid stent to reduce long-term stroke risks.

\section{ACKNOWLEDGEMENTS}

This study was supported by a Basic Science Research Program through the National Research Foundation of Korea (NRF) funded by the Ministry of Education, Science and Technology (No.2012R1A1B5000477).

\section{REFERENCES}

1. Pessin MS, Duncan GW, Mohr JP, Poskanzer DC. Clinical and angiographic features of carotid transient ischemic attacks. N Engl J Med 1977;296:358-62.

2. Norrving B, Nilsson B. Carotid artery occlusion: acute symptoms and long-term prognosis. Neurol Res 1981;3:229-36.

3. Jones HJ, Millikan CH. Temporal profile (clinical course) of acute carotid system cerebral infarction. Stroke 1976;7:64-71.

4. Meves SH, Muhs A, Federlein J, Buttner T, Przuntek H, Postert T. Recanalization of acute symptomatic occlusions of the internal carotid artery. J Neurol 2002;249:188-92.

5. Szabo K, Kern R, Gass A, Griebe M, Lanczik O, Daffertshofer M, et al. Early spontaneous recanalization following acute carotid occlusion. J Neuroimaging 2008;18:148-53.

6. Calleja S, De la Vega V, Llaneza JM, Lopez-Roger R, Gutierrez JM, Lahoz CH. Spontaneous recanalization of acute internal carotid artery occlusion. Ann Vasc Surg 2004;18:490-2.

7. Luitse MJ, Velthuis BK, Dauwan M, Dankbaar JW, Biessels GJ, Kappelle LJ, et al. Residual high-grade stenosis after recanalization of extracranial carotid occusion in acute ischemic stroke. Stroke 2015;46:12-5.

8. Adams HP Jr, Bendixen BH, Kappelle LJ, Biller J, Love BB, Gordon DL, et al. Classification of subtype of acute ischemic stroke. Definitions for use in a multicenter clinical trial. TOAST. Trial of org 10172 in acute stroke treatment. Stroke 1993;24:35-41.

9. Buslovich S, Hines GL. Spontaneous recanalization of chronic internal carotid artery occlusions: report of 3 cases. Vasc Endovascular Surg 2011;45:93-7.

10. Morris-Stiff G, Teli M, Khan PY, Ogunbiyi SO, Champ CS, Hibberd R, et al. Internal carotid artery occlusion: its natural history including recanalization and subsequent neurological events. Vas Endovascular Surg 2013;47:603-7.

11. Shah QA. Spontaneous recanalization after complete occlusion of the common carotid artery with subsequent embolic ischemic stroke. J Vasc Interv Neurol 2009;2:147-51.

12. Camporese G, Labropoulos N, Verlato F, Bernardi E, Ragazzi $\mathrm{R}$, Salmistraro G, et al. Benign outcome of objectively proven spontaneous recanalization of internal carotid artery occlusion. J Vasc Surg 2011;53:323-9.

13. Nam HS, Lee KY, Kim YD, Choi HY, Cho HJ, Cha MJ, et al. Failure of complete recanalization is associated with poor outcome after cardioembolic stroke. Eur J Neurol 2011;18:1171-8.

14. Qureshi AI, Siddiqui AM, Suri MF, Kim SH, Ali Z, Yahia AM, et al. Aggressive mechanical clot disruption and low-dose 
intra-arterial third-generation thrombolytic agent for ischemic stroke: a prospective study. Neurosurgery 2002;51:1319-27; discussion 1327-9.
15. Rha JH, Saver JL. The impact of recanalization on ischemic stroke outcome: a meta-analysis. Stroke 2007;38:967-73. 